\title{
On the Rate of Convergence of Continuous-Time Linear Positive Systems with Heterogeneous Time-Varying Delays
}

\author{
Hamid Reza Feyzmahdavian, Themistoklis Charalambous, and Mikael Johansson
}

\begin{abstract}
In this work, a set of conditions are presented for establishing exponential stability and bounds on the convergence rates of both general and positive linear systems with heterogeneous time-varying delays. First, a sufficient condition for delay-independent exponential stability of general linear systems is derived. When the time delays have a known upper bound, we present an explicit expression that bounds the decay rate of the system. We demonstrate that the best decay rate that our bound can guarantee can be easily found via convex optimization techniques. Finally, for positive linear systems, we show that the stability condition that we have developed is also necessary. The validity of the results is demonstrated via numerical examples.
\end{abstract}

\section{INTRODUCTION}

Many real-world processes, for example in economics, biology and communication networks, deal with physical quantities that cannot attain negative values. It is thus necessary that the state trajectories of dynamical models characterizing such processes are constrained within the positive orthant for all nonnegative initial conditions. Such systems are commonly referred to as positive systems [1]. Due to the ubiquity of positive systems and the wide variety of applications, the analysis and control of such systems has attracted considerable attention from the control community (see, for example, [2]-[8] and references therein).

Many processes that are described by positive systems are comprised of interconnected subsystems that exchange information. In reality, however, this information might not necessarily be transferred instantaneously and subsystems might not react instantaneously to changes in external stimulus. To describe the evolution of such systems, it is necessary to include models of time delays in the dynamical models. While general linear systems tend to be sensitive to time delays and typically become oscillatory or even unstable [9][14], positive linear systems have been shown to be insensitive to bounded time delays [15]-[17]. In other words, such systems are asymptotically stable if and only if the corresponding delay-free system is asymptotically stable.

Most of the results in the literature have studied asymptotic stability of positive linear systems. Nevertheless, there are processes and applications for which it is desirable that the system converges quickly enough to the equilibrium. However, quantitative stability measures, such as the decay rate, can be highly dependent on the magnitude of time delays. The exponential stability of positive linear systems

H. R. Feyzmahdavian, T. Charalambous, and M. Johansson are with ACCESS Linnaeus Center, School of Electrical Engineering, KTH-Royal Institute of Technology, SE-100 44 Stockholm, Sweden. Emails: \{hamidrez, themisc, mikaelj\}@kth.se. with constant time delays was recently investigated by Zhu et al. [18], where it is shown that the decay rate depends on the magnitude of the delays. Extensions, however, to the time-varying delay case are non-trivial. The main reason for this is that the results for constant delays rely on LyapunovKrasovskii functionals, which often lead to excessive conservativeness when the delays are time-varying.

In this paper, we develop a set of powerful conditions for establishing exponential stability of general and positive linear systems with heterogeneous time-varying delays. First, using a suitable Lyapunov-Razumikhin function, we derive a sufficient condition for delay-independent exponential stability of general linear systems. For the case when the time delays have a known upper bound, we present an explicit expression that bounds the decay rate of the system. We demonstrate that the best decay rate that our bound can provide, along with parameters for the associated LyapunovRazumikhin function, can be found via convex optimization techniques. Finally, for positive linear systems, we show that the stability condition that we have developed is also necessary.

The remainder of the paper is organized as follows. In Section II, we introduce the notation that will be used throughout the paper and review some required preliminaries that are useful for the development of the results in this paper. Section III gives a description of the problem under consideration. The main results of this paper are stated in Section IV. Illustrative examples are presented in Section V, justifying the validity and benefits of our results. Finally, concluding remarks are given in Section VI.

\section{Notation AND PRELIMINARIES}

\section{A. Notation}

Throughout the paper, vectors are written in bold lower case letters and matrices in capital letters. The non-negative orthant of the $n$-dimensional real space $\mathbb{R}^{n}$ is represented by $\mathbb{R}_{+}^{n}$. The $i^{t h}$ component of a vector $\boldsymbol{x}$ is denoted by $x_{i}$, and the notation $\boldsymbol{x} \geq \boldsymbol{y}$ implies that $x_{i} \geq y_{i}$ for all components $i$. For $A \in \mathbb{R}^{n \times n}, a_{i j}$ denotes the entry in row $i$ and column $j$, and $|A|$ denotes the matrix whose elements are $\left|a_{i j}\right|$ for all $i, j$. The matrix $\mathrm{A}$ is said to be Hurwitz if all its eigenvalues have negative real parts. The spectral abscissa of a matrix $A$ is the maximum real part of the eigenvalues of $A$ and is denoted by $\pi(A)$. Given a vector $\boldsymbol{v}>\mathbf{0}$, the weighted $l_{\infty}$ norm is defined by

$$
\|\boldsymbol{x}\|_{\infty}^{\boldsymbol{v}}=\max _{1 \leq i \leq n}\left|\frac{x_{i}}{v_{i}}\right| .
$$


For a real number $x$, the function $\operatorname{sgn}(x)$ is the signum function defined by

$$
\operatorname{sgn}(x)= \begin{cases}1, & \text { if } x>0 \\ 0, & \text { if } x=0 \\ -1, & \text { if } x<0\end{cases}
$$

\section{B. Preliminaries}

Next, we review the key definitions and results necessary for developing the main results of this paper. The following definition introduces non-negative and Metzler matrices.

Definition 1 A matrix $A \in \mathbb{R}^{n \times n}$ is called non-negative if $a_{i j} \geq 0$ for all $i, j$. It is called a Metzler matrix if $a_{i j} \geq 0$ for all $i \neq j$.

The next proposition provides some known properties of Metzler matrices. These conditions are useful when analyzing the stability of continuous-time positive linear systems.

Proposition 1 ( [19]) Let $A \in \mathbb{R}^{n \times n}$ be Metzler. Then, the following statements are equivalent.

(a) There exists a vector $\boldsymbol{v}>\mathbf{0}$ such that $A \boldsymbol{v}<\mathbf{0}$;

(b) A is Hurwitz.

The next definition introduces the logarithmic matrix norm.

Definition 2 ( [20]) For a square matrix $A \in \mathbb{R}^{n \times n}$, the logarithmic norm (also known as the measure) of $A$ is defined by

$$
\mu(A) \triangleq \lim _{s \rightarrow 0^{+}} \frac{\left\|I_{n}+s A\right\|-1}{s},
$$

where $I_{n}$ is the $n \times n$ identity matrix, and $\|\cdot\|$ is an induced matrix norm. Note that the logarithmic norm is not a matrix norm and that it may take negative values. For the weighted $l_{\infty}$ norm, the corresponding logarithmic norm of matrix $A$ is given by

$$
\mu_{\infty}^{\boldsymbol{v}}(A)=\max _{1 \leq i \leq n}\left\{a_{i i}+\sum_{j \neq i} \frac{1}{v_{i}}\left|a_{i j}\right| v_{j}\right\} .
$$

The Lyapunov-Razumikhin theorem is used extensively to analyze stability of systems with time delays. In what follows, we give a brief summary of the theorem. Consider the retarded functional differential equation

$$
\begin{aligned}
& \dot{\boldsymbol{x}}(t)=\boldsymbol{f}\left(t, \boldsymbol{x}\left(t-\tau_{1}(t)\right), \ldots, \boldsymbol{x}\left(t-\tau_{m}(t)\right)\right), \quad t \geq 0, \\
& \boldsymbol{x}(t)=\boldsymbol{\varphi}(t), \quad t \in[-\tau, 0],
\end{aligned}
$$

where $m \in \mathbb{N}, \boldsymbol{x}(t) \in \mathbb{R}^{n}$ is the state variable, $\tau_{i}(t)$ for $i=1, \ldots, m$ represent the time-varying delays satisfying $0 \leq \tau_{i}(t) \leq \tau$, and $\varphi(\cdot)$ is a given continuous vectorvalued function. The Lyapunov-Razumikhin theorem gives conditions for $\boldsymbol{x}(t)=\mathbf{0}$ to be uniformly asymptotically stable.

Proposition 2 ( [12]) Suppose $u, w: \mathbb{R}_{+} \rightarrow \mathbb{R}_{+}$are continuous non-decreasing functions, $v: \mathbb{R}_{+} \rightarrow \mathbb{R}_{+}$is a strictly increasing function, $u(s), v(s)$, and $w(s)$ are positive for $s>0$, and $u(0)=v(0)=0$. If there is a continuous functional $V$ such that:

i) $u(\|\boldsymbol{x}\|) \leq V(t, \boldsymbol{x}) \leq v(\|\boldsymbol{x}\|)$, for all $t \geq 0$ and for all $\boldsymbol{x} \in \mathbb{R}^{n}$;

ii) there exists a scalar $q>1$ such that the derivative of $V$ along the solution $\boldsymbol{x}(t)$ of (1) satisfies

$$
\begin{gathered}
\dot{V}(t, \boldsymbol{x}(t)) \leq-w(\|\boldsymbol{x}\|), \\
\text { if } V(t+\theta, \boldsymbol{x}(t+\theta)) \leq q V(t, x(t)), \theta \in[-\tau, 0],
\end{gathered}
$$

then, the time delay system (1) is uniformly asymptotically stable. If $u(s) \rightarrow \infty$ as $s \rightarrow \infty$, then (1) is also globally uniformly asymptotically stable.

Throughout this paper we will use the following concept of exponential stability.

Definition 3 The solution $\boldsymbol{x}(t)=\mathbf{0}$ of (1) is said to be globally exponentially stable if there exist positive reals $\alpha$ and $\beta$ such that for any initial condition $\varphi(\cdot)$, the solution $\boldsymbol{x}(t)$ of (1) satisfies

$$
\|\boldsymbol{x}(t)\| \leq \beta\left(\sup _{-\tau \leq s \leq 0}\|\boldsymbol{\varphi}(s)\|\right) e^{-\alpha t}, \quad \forall t \geq 0 .
$$

\section{Problem Statement}

Consider a linear system with time-varying (possibly) heterogeneous delays, given by

$$
\begin{aligned}
& \dot{x}_{i}(t)=\sum_{j=1}^{n} a_{i j} x_{j}(t)+\sum_{j=1}^{n} b_{i j} x_{j}\left(t-\tau_{j}^{i}(t)\right), \quad t \geq 0, \\
& x_{i}(t)=\varphi_{i}(t), \quad t \in[-\tau, 0], \quad i=1, \ldots, n,
\end{aligned}
$$

where $\boldsymbol{x}(t)=\left[x_{1}(t), \ldots, x_{n}(t)\right]^{T} \in \mathbb{R}^{n}$ is the state variable, $A=\left[a_{i j}\right] \in \mathbb{R}^{n \times n}, B=\left[b_{i j}\right] \in \mathbb{R}^{n \times n}$ are system matrices, and $\varphi(\cdot)=\left[\varphi_{1}(\cdot), \ldots, \varphi_{n}(\cdot)\right]^{T}$ is the continuous vectorvalued function specifying the initial state of the system. The delays $\tau_{j}^{i}(t)$ are assumed to be time-varying continuous functions with respect to $t$ and satisfy

$$
0 \leq \tau_{j}^{i}(t) \leq \tau, \quad \forall t \geq 0, i, j=1, \ldots, n,
$$

where $\tau$ is a positive constant. Note that $\tau_{j}^{i}(t)$ can be arbitrarily fast varying and no restriction on the derivative of $\tau_{j}^{i}(t)$ is imposed.

System $\mathcal{G}$ is said to be positive if for every non-negative initial condition $\varphi(\cdot) \in \mathbb{R}_{+}^{n}$, the corresponding state trajectory is non-negative, that is $\boldsymbol{x}(t) \geq \mathbf{0}$ for all $t \geq 0$. Positivity of $\mathcal{G}$ is readily verified using the following result.

Proposition 3 ( [17]) System $\mathcal{G}$ is positive if and only if $A$ is a Metzler matrix and $B$ is non-negative.

It should be stressed here that the non-negativity of the initial condition is essential for ensuring positivity of the state evolution of system $\mathcal{G}$. In other words, when $\varphi(\cdot) \in \mathbb{R}_{+}^{n}$ is not satisfied, $\boldsymbol{x}(t)$ may not stay in the positive orthant even if the conditions of Proposition 3 hold. 
While $\boldsymbol{x}=\mathbf{0}$ is clearly an equilibrium point of system $\mathcal{G}$, it is not necessarily stable. Moreover, the stability will, in general, depend on the magnitude and variation of the time delays. The main objectives of this paper is therefore to $(i)$ provide a sufficient condition for delay-independent exponential stability of the system $\mathcal{G}$ under heterogenous time-varying delays that is computationally simple to verify; and to $(i i)$ give explicit estimates of the decay rates of $\mathcal{G}$. Finally, as discussed in the introduction, positive linear systems are particular in the sense that they have been shown to be insensitive to bounded time delays [15]-[17]. Our final aim is hence to (iii) determine how the decay rate of such systems with bounded time-varying delays depends on the magnitude of the time delays.

\section{MAin Results}

Two important approaches in the stability analysis of time-delay systems are (a) Lyapunov-Krasovskii method, and (b) Lyapunov-Razumikhin method [12]-[14]. In [15], a necessary and sufficient condition for asymptotic stability of positive linear systems with constant delays has been derived by using a linear Lyapunov-Krasovskii functional. However, the Lyapunov-Krasovskii method often requires the time-varying delay $\tau(t)$ to satisfy conservative conditions on the rate of change of the delay, $\dot{\tau}(t)$. For investigating the exponential stability of positive linear systems with heterogenous time-varying delays, we employ a LyapunovRazumikhin approach which allows us to impose minimal restrictions on delays. In particular, we make use of the following Lyapunov-Razumikhin function

$$
V(\boldsymbol{x}(t))=\|\boldsymbol{x}(t)\|_{\infty}^{\boldsymbol{v}}
$$

where $v$ is a positive vector.

The following theorem is our first key result, which states a sufficient condition for delay-independent exponential stability of general linear systems with bounded time-varying delays.

Theorem 1 Suppose that there exists a vector $\boldsymbol{v}>\mathbf{0}$ such that

$$
a_{i i}+\left|b_{i i}\right|+\sum_{j \neq i} \frac{1}{v_{i}}\left(\left|a_{i j}\right|+\left|b_{i j}\right|\right) v_{j}<0,
$$

for $i=1, \ldots, n$. Let $\eta_{i}$ be the unique positive solution of the equation

$$
\eta_{i}+\left(\sum_{j=1}^{n} \frac{1}{v_{i}}\left|b_{i j}\right| v_{j}\right) e^{\eta_{i} \tau}=-\left(a_{i i}+\sum_{j \neq i} \frac{1}{v_{i}}\left|a_{i j}\right| v_{j}\right) .
$$

Then, system $\mathcal{G}$ is globally exponentially stable for any arbitrary bounded time-varying delays. In particular, every solution $\boldsymbol{x}(t)$ of $\mathcal{G}$ satisfies the condition

$$
\|\boldsymbol{x}(t)\|_{\infty}^{\boldsymbol{v}} \leq\|\boldsymbol{\varphi}\| e^{-\eta t}, \quad t \geq 0
$$

where $\eta=\min _{1 \leq i \leq n} \eta_{i}$, and

$$
\|\varphi\|=\sup _{-\tau \leq s \leq 0}\|\varphi(s)\|_{\infty}^{\boldsymbol{v}}
$$

Proof: See Appendix A.

Remark 1 Equation (3) has three parameters; namely, the positive vector $\boldsymbol{v}, \eta_{i}$, and the maximum allowable delay bound $\tau$. For any fixed $\tau \geq 0$, and any fixed $\boldsymbol{v}>\mathbf{0}$, (3) is a nonlinear equation with respect to $\eta_{i}>0$. From inequality (2), it is easily deduced that the left-hand side of the equation (3) is smaller than the right-hand side for $\eta_{i}=0$, and strictly monotonically increasing for $\eta_{i}>0$; therefore, (3) has always a unique solution $\eta_{i}$ which satisfies

$$
0<\eta_{i} \leq-\left(a_{i i}+\left|b_{i i}\right|+\sum_{j \neq i} \frac{1}{v_{i}}\left(\left|a_{i j}\right|+\left|b_{i j}\right|\right) v_{j}\right) .
$$

Note that $\eta_{i}$ is monotonically decreasing in $\tau$ for all $i$, and approaches zero as $\tau$ tends to infinity. Hence, Theorem 1 shows that the guaranteed decay rate slows down as the delays increase in magnitude.

Given a matrix $A \in \mathbb{R}^{n \times n}$, we define $A^{M}=\left[a_{i j}^{M}\right]$ to be

$$
a_{i j}^{M}=\left\{\begin{array}{rr}
a_{i i}, & i=j, \\
\left|a_{i j}\right|, & i \neq j .
\end{array}\right.
$$

Recalling the definition of $\mu_{\infty}^{v}$ (Definition 2), the stability condition (2) can now be rewritten as

$$
\mu_{\infty}^{v}\left(A^{M}+|B|\right)<0
$$

Remark 2 Since $A^{M}$ is Metzler and $|B|$ is non-negative, $A^{M}+|B|$ is a Metzler matrix. According to Proposition 1, Inequality (4) holds if and only if $A^{M}+|B|$ is Hurwitz.

Remark 3 It was shown in [11] that if $\mu(A)+\|B\|<0$, the linear system $\dot{\boldsymbol{x}}(t)=A \boldsymbol{x}(t)+B \boldsymbol{x}(t-\tau)$ with the constant delay $\tau \geq 0$ is asymptotically stable. Using the triangle inequality for the logarithmic norm [21, p. 41] gives

$$
\mu_{\infty}^{\boldsymbol{v}}\left(A^{M}+|B|\right) \leq \mu_{\infty}^{\boldsymbol{v}}(A)+\|B\|_{\infty}^{\boldsymbol{v}}
$$

where we used the fact that $\mu_{\infty}^{v}\left(A^{M}\right)=\mu_{\infty}^{v}(A)$ and $\mu_{\infty}^{\boldsymbol{v}}(|B|)=\|B\|_{\infty}^{\boldsymbol{v}}$. This indicates that for the weighted $l_{\infty}$ norm, the stability condition (4) is less conservative than that in [11].

Remark 4 In [10], the authors considered the linear timedelay system of the form

$$
\dot{x}_{i}(t)=a_{i i} x_{i}(t)+\sum_{j \neq i} a_{i j} x_{j}\left(t-\tau_{j}^{i}\right),
$$

where $i=1, \ldots, n$, and $\tau_{j}^{i}$ are non-negative constants for all $i, j$. It was proven in [10, Theorem 1] that if there exists a vector $\boldsymbol{v}>\mathbf{0}$ such that

$$
v_{i} a_{i i}+\sum_{j \neq i} v_{j}\left|a_{i j}\right|<0, \quad i=1, \ldots, n,
$$

then (5) is globally asymptotically stable. We now show that Theorem 1 coincides with this result and extends it to the case with time-varying delays. Define matrices

$$
A=\operatorname{diag}\left(a_{11}, \ldots, a_{n n}\right), B=\left[b_{i j}\right],
$$


where $b_{i j}=a_{i j}$ for $i \neq j$, and $b_{i i}=0$. According to Theorem 1 , if there exists a vector $\boldsymbol{v}>\mathbf{0}$ such that

$$
a_{i i}+\sum_{j \neq i} \frac{1}{v_{i}}\left|a_{i j}\right| v_{j}<0
$$

for all $i$, which is equivalent to (6), then the linear system (5) is globally exponentially stable for all bounded time delays. Note also that the impact of delays on the decay rate was missing in [10].

We will now show that the stability condition in Theorem 1 is not only sufficient but also necessary to guarantee exponential stability of positive linear systems with heterogenous bounded time-varying delays.

Theorem 2 Suppose that $A$ is a Metzler matrix and $B$ is a non-negative matrix. Then, the following statements are equivalent.

(a) $A+B$ is Hurwitz;

(b) There exists a vector $\boldsymbol{v}>\mathbf{0}$ such that $(A+B) \boldsymbol{v}<\mathbf{0}$;

(c) There exists a vector $\boldsymbol{v}>\mathbf{0}$ such that $\mu_{\infty}^{\boldsymbol{v}}(A+B)<0$;

(d) The positive system $\mathcal{G}$ is globally exponentially stable for all bounded time-varying delays.

Moreover, for any non-negative initial condition $\varphi(\cdot) \in \mathbb{R}_{+}^{n}$, the solution $\boldsymbol{x}(t)$ of $\mathcal{G}$ satisfies

$$
\|\boldsymbol{x}(t)\|_{\infty}^{\boldsymbol{v}} \leq\left(\sup _{-\tau \leq s \leq 0}\|\boldsymbol{\varphi}(s)\|_{\infty}^{\boldsymbol{v}}\right) e^{-\eta t}, \quad t \geq 0
$$

where $\eta=\min _{1 \leq i \leq n} \eta_{i}$, and $\eta_{i}$ is the unique positive solution of the following equation

$$
\eta_{i}+\left(\sum_{j=1}^{n} \frac{1}{v_{i}} b_{i j} v_{j}\right) e^{\eta_{i} \tau}=-\sum_{j=1}^{n} \frac{1}{v_{i}} a_{i j} v_{j} .
$$

Proof: See Appendix B.

According to Theorem 2, the positive linear system $\mathcal{G}$ is exponentially stable if and only if the corresponding system without delay is exponentially stable. In this case, the following set of linear inequalities in $v$,

$$
\left\{\begin{array}{l}
(A+B) \boldsymbol{v}<\mathbf{0} \\
\boldsymbol{v}>\mathbf{0}
\end{array}\right.
$$

is feasible. The above stability condition is a linear programming (LP) problem in $\boldsymbol{v}$ and, thus, can be verified numerically in polynomial time. Moreover, any feasible solution $\boldsymbol{v}$ to the above LP problem ensures that the solution $\boldsymbol{x}(t)$ of $\mathcal{G}$ is globally exponentially convergent to the origin with the rate $\eta=\min _{1 \leq i \leq n} \eta_{i}$, where $\eta_{i}$ satisfies (7). It is easily seen that the guaranteed decay rate depends on the choice of vector $\boldsymbol{v}$. Next, we will show that the best decay rate that our results can ensure, along with the associated vector $\boldsymbol{v}$ that parameterizes the Lyapunov-Razumikhin function can be found via convex optimization. To this end, we use the logarithmic change of variables $z_{i}=\log \left(v_{i}\right)$ (i.e., $v_{i}=e^{z_{i}}$ ) for $i=1, \ldots, n$. Then, the search for $\boldsymbol{v}$ can be formulated as $\operatorname{maximize}$
subject to:

$$
\begin{aligned}
& \eta \leq \eta_{i}, \\
& a_{i i}+b_{i i}+\sum_{j \neq i}\left(a_{i j}+b_{i j}\right) e^{z_{j}-z_{i}}<0, \\
& a_{i i}+\sum_{j \neq i} a_{i j} e^{z_{j}-z_{i}}+\sum_{j=1}^{n} b_{i j} e^{z_{j}-z_{i}+\eta_{i} \tau}+\eta_{i} \leq 0, \quad
\end{aligned}
$$

with (7) and (8) (in terms of $z$ ) being extra constraints. The optimization variables are the decay rate $\eta$ and the vector $\boldsymbol{z}=\left[z_{1}, \ldots, z_{n}\right]^{T}$. Since $a_{i j} \geq 0$ for all $i \neq j$ and $b_{i j} \geq 0$ for all $i, j$, the last two constraints in (9) are convex in $\eta$ and $z$. This implies that this is a convex optimization problem; hence, it can be efficiently solved.

Remark 5 The asymptotic stability of positive linear systems was investigated with constant delays in [15], and with time-varying delays in [16], [17]. Similar to Theorem 2, the stability conditions presented in [15]-[17] are necessary and sufficient, but for asymptotic stability. In this work, we show that the condition for exponential stability is also necessary and sufficient. Furthermore, the impact of delays on the decay rate of $\mathcal{G}$ has been missing in [15]-[17], whereas Theorem 2 provides an explicit bound on the decay rate that allows us to quantify the impact of delays on the decay rate.

\section{ILLUSTRATIVE EXAMPLES}

In this section, we illustrate our theoretical results by numerical examples. First, we demonstrate that Theorem 1 is less conservative than the stability criteria proposed in [22], [23]. Then, we explore our decay rate bound and demonstrate how the convex optimization formulation allows to find Lyapunov-Razumikhin functions with much stronger guarantees than alternative approaches.

Example 1: Consider the system described by $\mathcal{G}$ with

$$
A=\left[\begin{array}{cc}
-2 & -1 \\
0 & -2
\end{array}\right], B=\alpha\left[\begin{array}{ll}
0 & 1 \\
1 & 0
\end{array}\right],
$$

where $\alpha$ is a non-negative parameter. According to Theorem 1 and Remark 2, for global exponentially stability, it is sufficient that the matrix

$$
\left[\begin{array}{cc}
-2 & 1 \\
0 & -2
\end{array}\right]+\alpha\left[\begin{array}{ll}
0 & 1 \\
1 & 0
\end{array}\right]
$$

is Hurwitz which holds for $\alpha \leq 1.5616$.

For $\alpha=1$ and constant delay $\tau\left(\tau_{j}^{i}(t)=\tau\right.$ for all $\left.i, j\right)$, stability of (10) was studied in [22, Example 1] where it was shown that (10) is globally asymptotically stable if $\tau \leq 0.4212$. It was shown in [23, Example 3] that (10) is stable for arbitrary bounded time-varying delays, provided that $\alpha \leq 1.5322$. Hence, in this example, the result obtained by Theorem 1 is less conservative than those given in [22], [23]. Note also that the exponential stability of linear systems with heterogeneous time-varying delays were not considered in either [22] or [23]. 
Example 2: Consider system $\mathcal{G}$ with

$$
A=\left[\begin{array}{cc}
-6 & 2 \\
1 & -3
\end{array}\right], B=\left[\begin{array}{cc}
3 & 0 \\
0 & 0.5
\end{array}\right]
$$

The time-varying delays are given by $\tau_{1}^{1}(t)=5+\sin (t)$, and $\tau_{2}^{2}(t)=3+\cos (t)$. Obviously, one may choose $\tau=6$ as an upper bound on these delays. Moreover, $A$ is Metzler and $B$ is non-negative, so the system (11) is positive.

By Theorem 2, since $A+B$ is Hurwitz, (11) is globally exponentially stable, and hence the following set of inequalities must be fulfilled

$$
\left\{\begin{array}{c}
{\left[\begin{array}{cc}
-3 & 2 \\
1 & -2.5
\end{array}\right]\left[\begin{array}{l}
v_{1} \\
v_{2}
\end{array}\right]<\mathbf{0}} \\
v_{1}, v_{2}>0
\end{array}\right.
$$

As we have already discussed in Section IV, any feasible solution $\boldsymbol{v}$ of the above LP problem can be used to find a guaranteed rate of convergence of the system (11) by computing the associated $\eta$ in (7). One natural candidate for $\boldsymbol{v}$ can be found by considering the delay-free case (system $\mathcal{G}$ with $\left.\tau_{j}^{i}(t)=0\right)$. According to the Perron-Frobenius theorem for Metzler matrices [1, Theorem 17], if $A+B$ is Metzler and irreducible, then there exists an eigenvector $\boldsymbol{v}^{1}>\mathbf{0}$ such that

$$
(A+B) \boldsymbol{v}^{1}=\pi(A+B) \boldsymbol{v}^{1} .
$$

If $\pi(A+B)<0$, it is clear that vector $\boldsymbol{v}^{1}$ satisfies (12). For the positive system (11), $\pi(A+B)=-1.3139$, and the corresponding eigenvector is $\boldsymbol{v}^{1}=\left[\begin{array}{ll}0.7645 & 0.6446\end{array}\right]^{T}$. By using this solution together with $\tau=6$, the solutions to the nonlinear equation (7) can be obtained as $\eta_{1}=0.0583$, and $\eta_{2}=0.1957$. Thus, (11) is globally exponentially stable with decay rate $\eta=\min \{0.0583,0.1957\}=0.0583$. In particular,

$$
\|\boldsymbol{x}(t)\|_{\infty}^{\boldsymbol{v}^{1}} \leq\left(\sup _{-\tau \leq s \leq 0}\|\boldsymbol{\varphi}(s)\|_{\infty}^{\boldsymbol{v}^{1}}\right) e^{-0.0583 t}, \quad t \geq 0 .
$$

The left-hand side of Figure 1 gives the simulation results of the actual decay rate of the solution of $(11),\|\boldsymbol{x}(t)\|_{\infty}^{\boldsymbol{v}^{1}}$, and the theoretical upper bound $e^{-0.0583 t}$ when the initial condition is $\boldsymbol{\varphi}(t)=\boldsymbol{v}^{1}$. Of course, $\boldsymbol{v}^{1}$ is only one of the possible solutions of (12). Next, by solving the convex

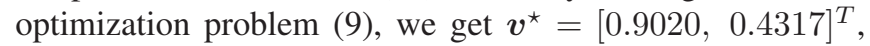
and $\eta^{\star}=0.0837$, which implies that the system (11) is globally exponentially stable with decay rate 0.0838 , and the solution $\boldsymbol{x}(t)$ satisfies

$$
\|\boldsymbol{x}(t)\|_{\infty}^{\boldsymbol{v}^{\star}} \leq\left(\sup _{-\tau \leq s \leq 0}\|\boldsymbol{\varphi}(s)\|_{\infty}^{\boldsymbol{v}^{\star}}\right) e^{-0.0838 t} .
$$

The right-hand side of Figure 1 gives the simulation results of $\|\boldsymbol{x}(t)\|_{\infty}^{\boldsymbol{v}^{\star}}$, and the theoretical upper bound $e^{-0.0838 t}$ when the initial condition is $\varphi(t)=\boldsymbol{v}^{\star}$.

We can see that the linear inequalities (12) do not help us in guiding our search for a vector $\boldsymbol{v}$ which guarantees a fast decay rate. In contrast, solving the convex optimization problem (9) finds the best $\eta^{\star}$ that our bound can guarantee along with the associated $\boldsymbol{v}^{\star}$.
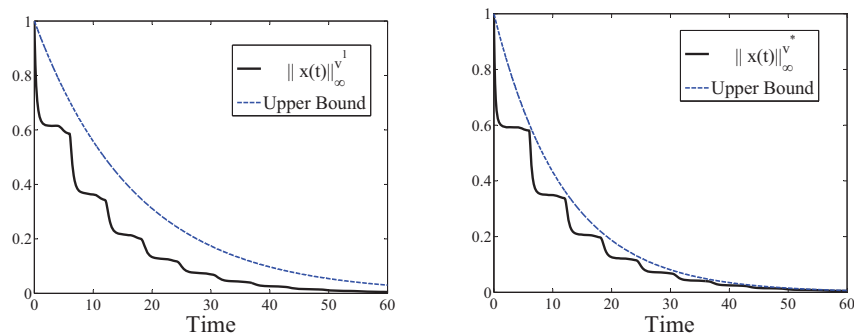

Fig. 1. Comparison of upper bounds and actual decay rates of the solution $\boldsymbol{x}(t)$ without (left) and with (right) convex optimization for the positive system described by (11) in Example 2.

\section{CONCLUSIONS}

In this paper, we have derived a set of easily verifiable conditions for establishing exponential stability of both general and positive linear systems with heterogeneous bounded time-varying delays. First, we derived a sufficient condition for delay-independent exponential stability of general linear systems. When the time delays have a known upper bound, an explicit expression that bounds the decay rate of the system was presented. The best decay rate that our bound can provide can be easily found via convex optimization techniques, as demonstrated in an illustrative example. Finally, for positive linear systems, we have shown that the stability condition developed is also necessary.

\section{APPENDIX}

\section{A. Proof of Theorem 1}

Consider $V(\boldsymbol{x}(t))=\|\boldsymbol{x}(t)\|_{\infty}^{\boldsymbol{v}}$ as a candidate Lyapunov function. This gives $\dot{V}(\boldsymbol{x}(t))=\frac{\operatorname{sgn}\left(x_{m}\right)}{v_{m}} \dot{x}_{m}(t)$ where $m$ is an index satisfying

$$
\frac{1}{v_{i}}\left|x_{i}(t)\right| \leq \frac{1}{v_{m}}\left|x_{m}(t)\right|
$$

for all $i$. Note that if (13) is satisfied by more than one value of $m$, we take $m$ as the one that has maximum $\frac{1}{v_{i}} \frac{\mathrm{d}\left|x_{i}\right|}{\mathrm{d} t}$. If still $m$ is not unique, then any of the remaining possibilities can be used. The time-derivative of $V(\boldsymbol{x}(t))$ along the trajectories of $\mathcal{G}$ is given by

$$
\begin{gathered}
\dot{V}(\boldsymbol{x}(t))=\frac{\operatorname{sgn}\left(x_{m}\right)}{v_{m}}\left\{\sum_{j=1}^{n} a_{m j} x_{j}(t)+\sum_{j=1}^{n} b_{m j} x_{j}\left(t-\tau_{j}^{m}(t)\right)\right\} \\
=\frac{1}{v_{m}}\left\{a_{m m}\left|x_{m}(t)\right|+\operatorname{sgn}\left(x_{m}\right) \sum_{j \neq m} a_{m j} x_{j}(t)\right. \\
\left.\quad+\operatorname{sgn}\left(x_{m}\right) \sum_{j=1}^{n} b_{m j} x_{j}\left(t-\tau_{j}^{m}(t)\right)\right\} \\
\leq \frac{1}{v_{m}}\left\{a_{m m}\left|x_{m}(t)\right|+\sum_{j \neq m}\left|a_{m j}\right|\left|x_{j}(t)\right|\right. \\
\left.\quad+\sum_{j=1}^{n}\left|b_{m j}\right|\left|x_{j}\left(t-\tau_{j}^{m}(t)\right)\right|\right\} \\
\leq \frac{1}{v_{m}}\left\{\left(a_{m m}+\sum_{j \neq m} \frac{1}{v_{m}}\left|a_{m j}\right| v_{j}\right)\left|x_{m}(t)\right|\right. \\
\left.\quad+\sum_{j=1}^{n}\left|b_{m j}\right|\left|x_{j}\left(t-\tau_{j}^{m}(t)\right)\right|\right\}
\end{gathered}
$$


where we used (13) to get the last inequality. In order to satisfy condition (ii) of the Lyapunov-Razumikhin theorem, Proposition 2, we are interested in the case

$$
\max _{t-\tau \leq s \leq t}\|\boldsymbol{x}(s)\|_{\infty}^{\boldsymbol{v}} \leq q\|\boldsymbol{x}(t)\|_{\infty}^{\boldsymbol{v}},
$$

for some $q>1$, which translates to

$$
\frac{1}{v_{i}}\left|x_{i}(s)\right| \leq q\|\boldsymbol{x}(t)\|_{\infty}^{\boldsymbol{v}}, \quad s \in[t-\tau, t], i=1, \ldots, n .
$$

Thus, since $\|\boldsymbol{x}(t)\|_{\infty}^{\boldsymbol{v}}=\frac{1}{v_{m}}\left|x_{m}(t)\right|$, (14) can be written as

$$
\begin{aligned}
\dot{V}(\boldsymbol{x}(t)) \leq\left(a_{m m}\right. & \sum_{j \neq m} \frac{1}{v_{m}}\left|a_{m j}\right| v_{j}+ \\
& \left.+q \sum_{j=1}^{n} \frac{1}{v_{m}}\left|b_{m j}\right| v_{j}\right)\|\boldsymbol{x}(t)\|_{\infty}^{\boldsymbol{v}} .
\end{aligned}
$$

Let $\eta_{i}$ be a solution of the equation

$$
\eta_{i}+\left(\sum_{j=1}^{n} \frac{1}{v_{i}}\left|b_{i j}\right| v_{j}\right) e^{\eta_{i} \tau}=-\left(a_{i i}+\sum_{j \neq i} \frac{1}{v_{i}}\left|a_{i j}\right| v_{j}\right),
$$

for $i=1, \ldots, n$. Note that, according to Remark 1 , the nonlinear equation (16) has a unique positive solution. Define $\eta=\min _{1 \leq i \leq n} \eta_{i}$. Since the left-hand side of (16) is monotonically increasing in $\eta_{i}$, we have

$$
\eta+\left(\sum_{j=1}^{n} \frac{1}{v_{i}}\left|b_{i j}\right| v_{j}\right) e^{\eta \tau} \leq-\left(a_{i i}+\sum_{j \neq i} \frac{1}{v_{i}}\left|a_{i j}\right| v_{j}\right),
$$

for all $i$. Set $q=e^{\eta \tau}$. Substituting $q$ into (15) and using (17) yields

$$
\dot{V}(\boldsymbol{x}(t)) \leq-\eta V(\boldsymbol{x}(t)) .
$$

Therefore, according to the Lyapunov-Razumikhin theorem, system $\mathcal{G}$ is globally asymptotically stable. Integrating both sides of (18) follows that $V(\boldsymbol{x}(t)) \leq e^{-\eta t} V(\boldsymbol{x}(0))$, and hence

$$
\|\boldsymbol{x}(t)\|_{\infty}^{\boldsymbol{v}} \leq\left(\sup _{-\tau \leq s \leq 0}\|\boldsymbol{\varphi}(s)\|_{\infty}^{\boldsymbol{v}}\right) e^{-\eta t}, \quad t \geq 0 .
$$

This completes the proof of the theorem.

\section{B. Proof of Theorem 2}

$(a) \Leftrightarrow(b) \Leftrightarrow(c)$ : Since $A$ is Metzler and $B$ is nonnegative, $A+B$ is a Metzler matrix. Hence, the equivalence of (a) and (b) follows directly from Proposition 1. Moreover, from Definition 2, we have

$$
\mu_{\infty}^{v}(A+B)=\max _{1 \leq i \leq n}\left\{a_{i i}+b_{i i}+\sum_{j \neq i} \frac{1}{v_{i}}\left(a_{i j}+b_{i j}\right) v_{j}\right\} .
$$

Therefore, (b) implies (c), and vice versa.

$(c) \Rightarrow(d)$ : According to Theorem $1, \mu_{\infty}^{v}\left(A^{M}+|B|\right)<0$ is a sufficient condition for exponentially stability of system $\mathcal{G}$. Since $A$ is Metzler and $B$ is non-negative, $A^{M}=A$ and $|B|=B$. Thus, (c) implies (d). $(d) \Rightarrow(a)$ Assume that the positive system $\mathcal{G}$ is exponentially stable for all bounded time delays. Particularly, let $\tau_{j}^{i}(t)=0$. Then, the linear system $\dot{\boldsymbol{x}}(t)=(A+B) \boldsymbol{x}(t)$ is globally exponentially stable. The conclusion follows from [6, Theorem 2.11].

\section{REFERENCES}

[1] L. Farina and S. Rinaldi, Positive Linear Systems: Theory and Applications. John Wiley and Sons, New York, 2000.

[2] P. D. Leenheer and D. Aeyels, "Stabilization of positive linear systems," Systems and Control Letters, vol. 44, pp. 259-271, 2001.

[3] M. A. Rami and F. Tadeo, "Controller synthesis for positive linear systems with bounded controls," IEEE Transactions on Circuits and Systems II, vol. 54, no. 2, pp. 151-155, Feb. 2007.

[4] F. Knorn, O. Mason, and R. Shorten, "On linear co-positive Lyapunov functions for sets of linear positive systems," Automatica, vol. 45, no. 8, pp. 1943-1947, 2009.

[5] T. Tanaka and C. Langbort, "KYP lemma for internally positive systems and a tractable class of distributed $\mathcal{H}_{\infty}$ control problems," American Control Conference (ACC), pp. 6238-6243, 2010.

[6] W. M. Haddad, V. Chellaboina, and Q. Hui, Nonnegative and Compartmental Dynamical Systems. Princeton, New Jersey, 2010.

[7] A. Rantzer, "Distributed control of positive systems," 50th IEEE Conference on Decision and Control and European Control Conference (CDC-ECC), pp. 6608-6611, 2011.

[8] C. Briat, "Robust stability analysis of uncertain linear positive systems via integral linear constraints: $L_{1}$ - and $L_{\infty}$-gain characterizations," 50th IEEE Conference on Decision and Control and European Control Conference (CDC-ECC), pp. 6337-6342, 2011.

[9] R. D. Driver, Ordinary and Delay Differential Equations. Springer, New York, 1977.

[10] R. Lewis and B. Anderson, "Necessary and sufficient conditions for delay-independent stability of linear autonomous systems," IEEE Transactions on Automatic Control, vol. 25, no. 4, pp. 735-739, Aug. 1980.

[11] T. Mori, N. Fukuma, and M. Kuwahara, "On an estimate of the decay rate for stable linear delay systems," Int. J. Control, vol. 36, pp. 95-97, 1982.

[12] J. K. Hale and S. M. V. Lunel, Introduction to Functional Differential Equations. Springer, New York, 1993.

[13] S. I. Niculescu, Delay effects on stability. A robust control approach. Springer-Verlag, 2001.

[14] K. Gu, V. Kharitonov, and J. Chen, Stability of Time-Delay Systems. Birkhauser, 2003.

[15] W. Haddad and V. Chellaboina, "Stability theory for non-negative and compartmental dynamical systems with time delay," Syst. Control Lett., vol. 51, no. 5, pp. 355-361, 2004.

[16] M. Ait Rami, "Stability analysis and synthesis for linear positive systems with time-varying delays," in Positive systems - Proceedings of the 3rd Multidisciplinary International Symposium on Positive Systems: Theory and Applications (POSTA), pp. 205-216, 2009.

[17] X. Liu, W. Yu, and L. Wang, "Stability analysis for continuous-time positive systems with time-varying delays," IEEE Transactions on Automatic Control, vol. 55, no. 4, pp. 1024-1028, April 2010.

[18] S. Zhu, Z. Li, and C. Zhang, "Exponential stability analysis for positive systems with delays," Control Theory Applications, IET, vol. 6, no. 6, pp. 761-767, 2012.

[19] A. Berman and R. J. Plemmons, Nonnegative Matrices in Mathematical Sciences. Academic Press, New York, 1979.

[20] M. Vidyasagar, "On matrix measures and convex Lyapunov functions," Journal of Mathematical Analysis and Applications, vol. 62, pp. 90103, 1982.

[21] W. A. Coppel, Stability and Asymptotic Behavior of Differential Equations. D. C. Heath, Boston, 1965.

[22] P.-L. Liu and T.-J. Su, "Robust stability of interval time-delay systems with delay-dependence," Syst. Control Lett., vol. 33, pp. 231-239, 1998.

[23] A. Zevin and M. Pinsky, "Delay-independent stability conditions for time-varying nonlinear uncertain systems," IEEE Transactions on Automatic Control, vol. 51, no. 9, pp. 1482-1486, Sept. 2006. 